\title{
Minimal contrast in artificial word associations $^{1}$
}

CHARLES A. PERFETTI

UNIVERSITY OF PITTSBURGH

The minimal contrast hypothesis was tested with artificial words. In a modified paired-associates task, Ss first learned "semantic" features of artificial words, then gave associations to each word. Associations tended to be words which contrasted with the stimulus on only one feature, thus supporting the minimal contrast hypothesis for artificial words.

The notion that words can be decomposed into morphemic semantic features has been suggested from diverse linguistic (Katz \& Fodor, 1963), anthropological (Romney \& D'Andrade, 1964), and psychological (Osgood, 1966) sources. The concomitant suggestion that such semantic features have a role in determining word association has also been made (Saporta, 1959; McNeill, 1966). In brief, the semantic featural analysis of word association supposes the following: Two words BOY and GIRL share a large number of semantic features such as (human), (young), etc. and contrast on a minimum number (sex). Because the number of contrasting features is minimal, BOY will be more likely to evoke GIRL as an associate than WOMAN, which contrasts with it on an additional feature dimension, (age). Of course a feature analysis is not needed to account for associations that can be explained by frequency of cooccurrence in the language, as is the case with BOYGIRL. The question is whether a feature analysis is a useful way to describe the semantic aspects of association.

The present experiment was designed to provide a sufficiency test for this minimal contrast hypothesis under circumstances which provide control over the semantic features but which do not allow specifiable competing sources of association. The hypothesis receives the following operationalization in the present experiment: If Ss acquire semantic features for artificial concept words and then are required to associate to the artificial words, responses will tend to be words which are minimal contrasts in terms of the acquired features.

\section{Method}

Materials. Artificial words were chosen from a specially constructed list of 18 two-syllable words, each having five letters without repetition in a consonant-vowel sequence of C VCVC. No two words had the same consonant in the same position. The six words randomly selected from this balanced list were MAPOK, JUWEN, NYJIB, KEBYR, POGUL, and VIKAF.

The features imposed on the artificial words were chosen so as to minimize the likelihood that any combination of features could be uniformly interpreted as constituting an English word. A second selection criter- ion was that the features be pairs of adjective contrasts. The three feature dimensions finally selected were (tangible-intangible), (wet-dry), and (public-private). In the assignment of features to words, each feature occurred equally often and in combination with every other feature. Two examples from the resulting list of concept words are VIKAF: (tangible), (wet), (public); and MAPOK: (intangible), (dry), (private).

Subjects and Procedure.Forty female undergraduates at the University of Michigan served in the experiment. The Ss participated individually on four tasks, after first becoming familiar with a standardized pronunciation of each artificial word: (1) S first learned the feature composition of the artificial words in a modified paired-associates task. The $S$ saw a card with a feature question (e.g., MAPOK: WET or DRY?), anticipated which feature was correct, and then saw the correct answer in the form: A MAPOK is DRY. Thus, there were 18 anticipation trials (6 words $x 3$ features) for each complete trial block. The criterion for learning was two complete trial blocks without an error. The procedure demanded that $S$ pronounce every artificial word six times for each complete trial block, thus assuring fluency with the words prior to subsequent tasks. (2) Immediately after criterion attainment, $S$ was asked to name all the features of each word. Seven Ss who failed on this task were discarded for the purpose of further data analysis. The final tasks were (3) free recall of the artificial words, and (4) "free" association to the artificial words with the restriction that the response be another artificial word.

\section{Results}

Learning. The mean number of trial blocks to criterion was 6.94 , with $\mathrm{SD}=3.55$. The difference in acquisition rate for individual words reached borderline significance in a one-way analysis of variance for nonindependent samples $(F=2.53, p<.05)$. However, no individual comparisons were significant according to a Newman-Keuls comparison test.

Free recall. The main interest in the recall data was the order of emission of items, in particular whether the dimensional structure of the concept words would be reflected by the joint occurrence of items with common features. That is, we were interested in whether we would observe semantic clustering of the artificial words.

The following statements summarize the cluster analysis: (1) The tendency for successively recalled words to share at least one feature did not significantly exceed chance $(p>.20)$. (2) No differences were found a mong the frequencies of occurrence of the various features and 
feature combinations; i.e., words with common features which were successively recalled were equally likely to be (tangible) words or (dry) words or any possible combination of features. (3) There was no minimal contrast clustering. The frequency of successively recalled words contrasting on only one feature did not exceed chance.

Association. The data from the association taskgave strong support for the minimal contrast hypothesis: (1) The mean percent MCR (minimal contrast response) for the six stimuli was 54 , significantly $(p<.001)$ above the chance level of 40 . (2) Every stimulus evoked an MCR as its most frequent response. (3) Every stimulus evoked MCR more frequently than expected by chance, with the percent MCR ranging from 48 for the stimulus KEBYR to 64 for JUWEN.

Figure 1 shows the general relationship between the number of shared features between associates and the observed probability of their association. The probabilities have been corrected for unequal chance levels. (That is, the observed proportions have been adjusted so that they may be compared with the same chance level.) The minimal contrast probability (two shared features) shows a sharp rise compared with associates sharing zero or one feature.

The generality of the main result is supported by the results of two additional analyses. First, the differences among stimuli in their probabilities of evoking MCR were found to be nonsignificant $\left(X^{2}=1.39, \mathrm{df}=5, \mathrm{p}>.90\right.$ ). Secondly, the differences among words in their emission as responses were also nonsignificant $\left(X^{2}=1.76\right.$, df $=\mathrm{S}, \mathrm{p}>.80$ ).

Finally, the possibility that a specific feature dimension might have provided a pronounced source of contrast was tested by two separate $\chi^{2}$ tests, one for associations involving double contrasts and one for single contrasts.

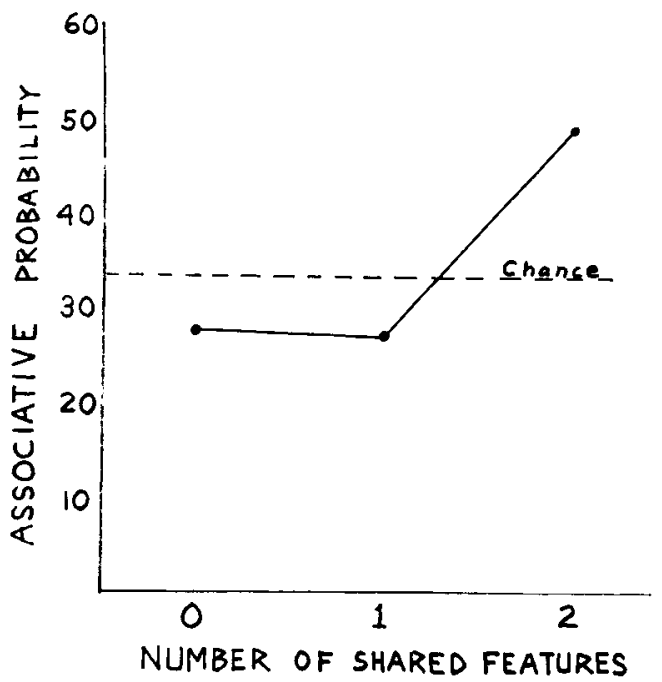

Fig. 1. Associative probability as a function of number of shared features between associates.
In neither case was there a significant tendency for one feature dimension rather than another to provide the source of contrast for an association. (Probabilities associated with the two $\chi^{2}$ values were .30 and .70 , respectively, for double and single contrasts.)

\section{Discussion}

In the main results the support for the minimal contrast hypothesis is statistical in that it depends upon comparison with chance level and, consequently, upon the assumption that associative probabilities were equal for all word pairs prior to feature assignment. Control for the possibility that this assumption is unwarranted came from another experiment (Perfetti, 1967, Experiment 3) which employed exactly the same words but reassigned features, with the result that every stimulus word had a different MCR associate from the one that was an MCR associate in the present experiment. For example, in the present experiment, the minimal contrasts for POGUL were JUWEN and MAPOK, while in the control experiment KEBYR was the minimal contrast for POGUL. The association results of the control experiment were essentially the same as for the present experiment, thus giving substantial support to the validity of the minimal contrast effect.

The specific locus of the effect remains unclear, however. The following hypotheses cannot confidently be ruled out on the basis of the present experiment: (1) Features are actively matched or contrasted by $S$ during association. (2) Words are "paired" prior to association, presumably during learning. (3) Ss use a single feature of the stimulus to mediate the response. Other data analyses on this experiment and two others (Perfetti, 1967) tend to make (3) slightly less plausible than (1) or (2).

\section{References}

KATZ, J. J., \& FODOR, J. A. The structure of a semantic theory. Language, 1963, 39, 170-210. Reprinted in J. A. Fodor and J. J. Katz, (Eds.), The structure of language: readings in the philosophy of language. New Jersey: Prentice-Hall, Inc., 1964, pp. 479-518.

McNEILL, D. A study of word association. J. verb. Learn. verb. Behav., $1966,5,548-557$.

OSGOOD, C. E. Speculation on the structure of interpersonal intentions. Tech. Rep. No. 39. Institute of Communications Research, Urbana, Ill., 1966.

PERFETTI, C. A. A semantic featural approach to meaning similarity and association. Rep. No. 18, Language Development Program, Center for Human Growth and Development, University of Michigan.

ROMNEY, A. K., \& D'ANDRADE, G. G. Cognitive aspects of English kin terms. Amer. Anthrop. (spec. pub.), 1964, 66, no. 3, 146-170.

SAPORTA, $\mathrm{S}$. Linguistic structure as a factor and as a measure in word association. In J. J. Jenkins (Ed.), A ssociative processes in verbal behavior; a report of the Minnesota Conference, Minneapolis: Univer. of Minnesota, Dept. of Psych., 1959.

Note

1. This paper describes one of five experiments previously reported in detail in Report Number 18 of the Language Development Program, Center for Human Growth and Development, University of Michigan, which was the author's Ph. D dissertation submitted to the University of Michigan in April, 1967. 